This item was submitted to Loughborough's Research Repository by the author.

Items in Figshare are protected by copyright, with all rights reserved, unless otherwise indicated.

\title{
Anomalous cross-field diffusion in a magnetic trap
}

PLEASE CITE THE PUBLISHED VERSION

http://dx.doi.org/10.1103/PhysRevE.90.062117

\section{PUBLISHER}

(C) American Physical Society

\section{VERSION}

VoR (Version of Record)

\section{PUBLISHER STATEMENT}

This work is made available according to the conditions of the Creative Commons Attribution-NonCommercialNoDerivatives 4.0 International (CC BY-NC-ND 4.0) licence. Full details of this licence are available at: https://creativecommons.org/licenses/by-nc-nd/4.0/

\section{LICENCE}

CC BY-NC-ND 4.0

\section{REPOSITORY RECORD}

Savel'ev, Sergey, and Fabio Marchesoni. 2019. "Anomalous Cross-field Diffusion in a Magnetic Trap". figshare. https://hdl.handle.net/2134/18512. 


\title{
Anomalous cross-field diffusion in a magnetic trap
}

\author{
Sergey E. Savel'ev ${ }^{1}$ and Fabio Marchesoni ${ }^{2}$ \\ ${ }^{1}$ Department of Physics, Loughborough University, Loughborough LE11 3TU, United Kingdom \\ ${ }^{2}$ Dipartimento di Fisica, Università di Camerino, I-62032 Camerino, Italy
}

(Received 14 May 2014; published 10 December 2014)

\begin{abstract}
We numerically simulated the diffusion of a charged Brownian particle confined to a plane under the action of an orthogonal magnetic field with intensity depending on the distance from a center. Despite its apparent simplicity, this system exhibits anomalous diffusion. For positive field gradients, radial and angular dynamics are asymptotically subdiffusive, with exponents given by simple analytical expressions. In contrast, when driven by a weakly decaying field, the particle attains normal diffusion only after exceedingly long superdiffusive transients. These mechanisms can be related to Bohm diffusion in magnetized plasmas.
\end{abstract}

DOI: 10.1103/PhysRevE.90.062117

PACS number(s): 05.40.-a, 52.30.-q, 94.30.-d

\section{INTRODUCTION}

In plasma physics the term anomalous diffusion traditionally refers to situations where the diffusion process itself is normal, i.e., the particle mean-square displacement (m.s.d) grows linearly with time, but the relevant diffusion constant exhibits peculiar (and often unexpected) properties. A well-known example is the so-called Bohm diffusion [1], whereby excess cross-field diffusion was observed in magnetized plasmas under diverse experimental conditions [2]. In such experiments the plasma diffusion constant was often reported to decrease with the magnetic field slower than predicted by classical transport theory. Another example, also related to the present work, is the suppression of magnetodiffusion of electrons across a classical Lorentz gas of circular elastic scatterers [3]. Anomalous magnetodiffusion in the sense of a nonlinear asymptotic time dependence of particle m.s.d. [4,5] has been reported and extensively investigated in the more recent literature, mostly in connection with magnetotransport on some sort of periodic $[6,7]$ or weakly disordered substrate [8-11].

To investigate conditions reminiscent of Bohm diffusion we simulated the lateral diffusion of a charged Brownian particle constrained to move in a plane threaded through by an inhomogeneous magnetic field. In the stylized device of Fig. 1 the particle is free to diffuse in a homogeneous medium (at rest and in equilibrium with constant temperature) confined to the $x-y$ plane, only driven by the component of a magnetic field orthogonal to the plane. The modulus of the orthogonal components of the magnetic field is constant in time, but varies monotonically with the distance from the center; the radial component of the magnetic field does not affect the planar diffusion and, therefore, can be neglected. This is a possibly oversimplified instance of a wide class of magnetic traps one encounters when dealing with problems as diverse as plasma confinement [12], cosmic rays propagation [13], and magnetic tweezers [14]. Nevertheless, the numerical investigation of cross-field diffusion in the magnetic trap of Fig. 1 revealed a surprising property: the m.s.d of the particle from the center of the trap grows with time according to an asymptotic power law with anomalous exponent that depends on the radial gradient of the magnetic field; for positive field gradients anomalous diffusion holds asymptotically with exponents smaller than one (subdiffusion), whereas for negative field gradients the exponents are larger than one and anomalous diffusion is restricted to (possibly long) transients (superdiffusion) [4,5]. At the best of our knowledge, anomalous diffusion had never been detected in a low-dimensional Hamiltonian system coupled to an equilibrium heat bath.

In classical transport theory any time an anomalous diffusion process is encountered, investigators try to classify the underlying microscopic mechanism responsible for it, according to a few distinct paradigmatic frameworks [15]. Among the best established are continuous-time random walks, diffusion on fractal topology, and fractional Brownian motion [16]. Such schemes assume either many-body dynamics in a constrained homogeneous space (like single files of Brownian particles [17]), or single-particle dynamics coupled to a inhomogeneous trapping substrate (like random confining geometries [18] or chaotic islands in phase space [19]), or highly non-Markovian noise sources [20]. Contrary to all of this, the stationary diffusion process reported here just involves a particle with spatially dependent chirality, confined to a two-dimensional (2D) variety and subjected to Gaussian $\delta$-correlated thermal fluctuations.

As in the Bohr-van Leeuwen theorem [12], the inhomogeneous potential vector associated with a space-dependent magnetic field does not suffice, alone, to establish a nonuniform equilibrium distribution of the diffusing charged particle. Due to the interplay of equilibrium fluctuations and damping introduced to model the action of the heat bath, the particle diffuses without geometric constraints and, therefore, cannot be stably trapped by a magnetic inhomogeneity. However, as the radial diffusion of the injected charged particles gets anomalously suppressed, depending on the field radial gradient, the magnetic-field lines sketched in Fig. 1 can operate as a metastable stochastic trap, where particles can sojourn for arbitrarily long periods of time.

\section{MODEL}

The position $\mathbf{r}=(x, y)$ of a charged Brownian particle of mass $m$ and charge $q$, confined to the $x-y$ plane, and subject to an inhomogeneous magnetic field $\mathbf{B}=B(r) \hat{\mathbf{z}}$, evolves in time according to the Langevin equations [21]

$$
\begin{aligned}
& \ddot{x}=-\gamma \dot{x}+\Omega \dot{y}+\sqrt{D_{0}} \xi_{x}(t), \\
& \ddot{y}=-\gamma \dot{y}-\Omega \dot{x}+\sqrt{D_{0}} \xi_{y}(t),
\end{aligned}
$$


(a)
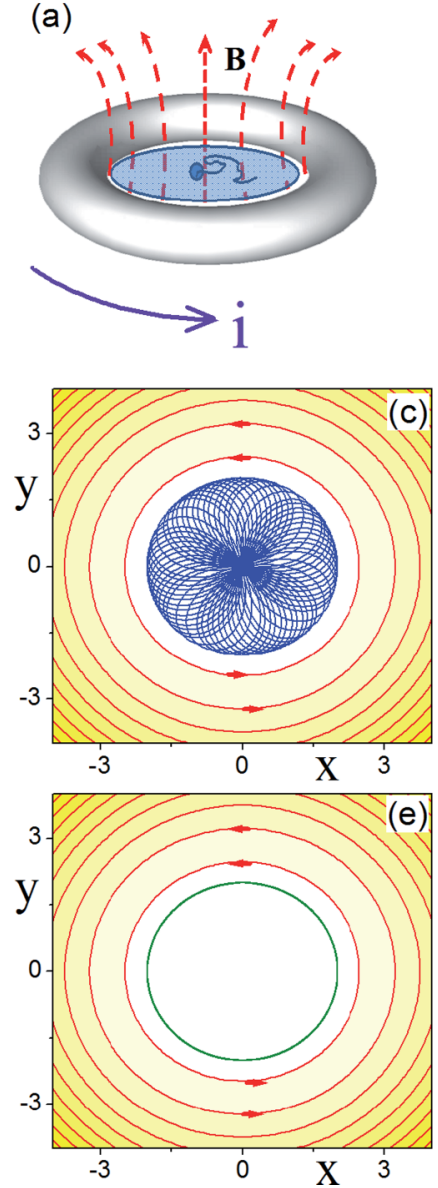
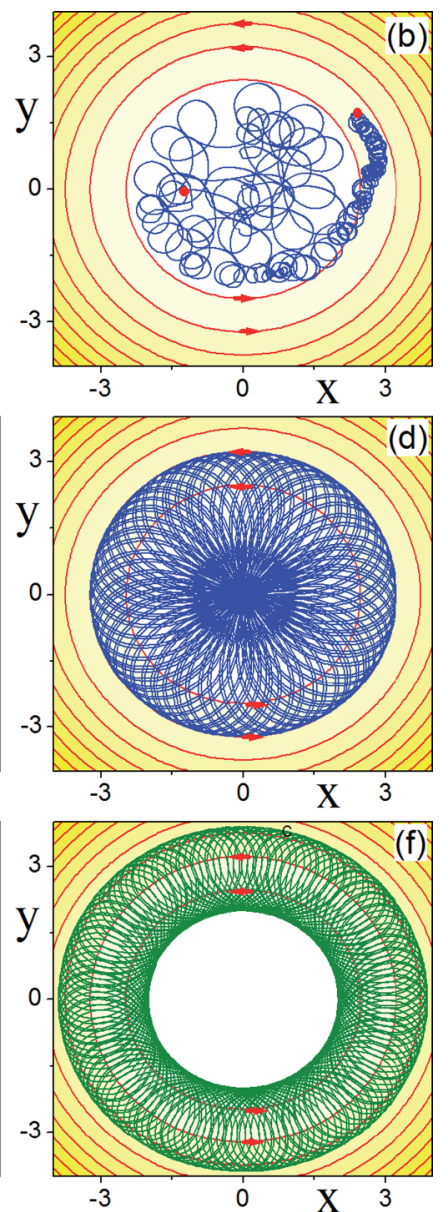

FIG. 1. (Color online) Sketch of a 2D magnetic trap with orthogonal magnetic field, $\mathbf{B}=B(r) \hat{\mathbf{z}}$ (vertical arrows) (a), and rotational potential vector $\mathbf{A}(r)$ (circular field lines) (b)-(f). Panel (a): a charged Brownian particle is confined to the plane region encircled by a ring traversed by constant electric current. Panel (b): trajectory sample obtained by integrating the Langevin equations (1) with $q=m=1, k T=1, \gamma=0.1$, and cyclotron frequency of Eq. (2) with $\Omega_{0}=\Omega_{r}=r_{0}=1$ and $n=2$. The end points are marked by dots. Panels (c)-(f): deterministic trajectories, $\gamma=0$, with initial conditions (c) $\mathbf{r}(0)=(0,0), \mathbf{v}(0)=(0,3),(\mathrm{d}) \mathbf{r}(0)=(0,0), \mathbf{v}(0)=(0,10)$, (e) $\mathbf{r}(0)=(2,0), \mathbf{v}(0)=(0,10)$, and (f) $\mathbf{r}(0)=(2,0), \mathbf{v}(0)=(0,-10)$. The inner and outer radial bounds, $r_{m}$ and $r_{M}$, coincide with the analytical estimates given in the text.

where $\boldsymbol{\Omega}=q \mathbf{B} / m$ is the corresponding space-dependent cyclotron frequency, $D_{0}=\gamma k T / m \equiv \gamma v_{\text {th }}^{2}$ with $T$ and $\gamma$ the temperature and viscous constant of the equilibrium suspension medium, and $\xi_{i}(t)$ with $i, j=x, y$ are Gaussian white noises with correlation functions $\left\langle\xi_{i}(t)\right\rangle=0$ and $\left\langle\xi_{i}(t) \xi_{j}(0)\right\rangle=$ $2 \delta_{i j} \delta(t)$. The field $\mathbf{B}$ exerts on the particle an external torque, $\tau_{\Omega}=(\Omega \dot{y},-\Omega \dot{x})$, which bends its mean-free path into a circular arc of Larmor radius $R_{\Omega}=v_{\mathrm{th}} /|\Omega|$ and length $l_{\Omega}=$ $v_{\text {th }} / \gamma, v_{\text {th }}$ denoting the thermal velocity $v_{\text {th }}=\sqrt{k T / m}$.

As for the spatial dependence of the magnetic field, $B(r)$ was modeled by a polynomial function of the distance $r=$ $\sqrt{x^{2}+y^{2}}$ from the axis, namely, $B(r)=B_{0}+B_{r}\left(r / r_{0}\right)^{n}$ with $B_{0}, B_{r} \geqslant 0$ (see sketches in Fig. 1). Accordingly, the cyclotron

frequency $\Omega$ in Eq. (1) is also a radial function,

$$
\Omega(r)=\Omega_{0}+\Omega_{r}\left(r / r_{0}\right)^{n},
$$

with $\Omega_{0}=q B_{0} / m$ and $\Omega_{r}=q B_{r} / m$. The radial gradients of $B(r)$ and $\Omega(r)$ are controlled by the characteristic trapping length $r_{0}$ and the exponent $n$. Unless stated otherwise, we assume that $n>0$; for $n \rightarrow 0$ the case of uniform $\mathbf{B}$ is recovered and $\Omega(r)=\Omega_{0}+\Omega_{r}$. By making use of the polar coordinates $(r, \phi)$, with $x=r \cos \phi$ and $y=r \sin \phi$, one sees immediately that the corresponding vector potential $\mathbf{A}$ lies in the $x-y$ plane with zero radial component and counterclockwise tangential component $A_{\phi}(r)=r\left[B_{0} / 2+B_{1}\left(r / r_{0}\right)^{n} /(n+2)\right]$ [see Fig. 1(b)].

The dynamics described by Eq. (1) is strongly influenced by the equilibrium heat bath, which acts upon the particle by means of the damping and fluctuation terms. For the sake of a comparison with the results reported below, we recall that in the noiseless regime, $\gamma \equiv 0$ (no heat-bath coupling), the system has two constants of motion, namely, the kinetic energy, $E=m\left(\dot{x}^{2}+\dot{y}^{2}\right) / 2(\mathbf{B}$ does no work, being orthogonal to the particle trajectory), and the canonical momentum associated with the polar angle $\phi, p_{\phi}=m r^{2} \dot{\phi}+q r A_{\phi}(r)$ [Eqs. (1) with $\gamma=0$ are invariant under rotations around the $z$ axis]. As a consequence, the system is integrable and its trajectories are nonchaotic [a few examples are displayed in panels (c)-(f) of Fig. 1]. In particular, the orbits of a particle with initial conditions $\mathbf{r}(0)=0$ and $\dot{\mathbf{r}}(0)=\overline{\mathbf{v}}$ are in general open and bound inside a circle of radius $r_{M}$, where $r_{M}$ is the solution of the equation $(q / m) A_{\phi}\left(r_{M}\right)=\bar{v}$, with $\bar{v}=|\overline{\mathbf{v}}|$ a constant [panels (c) and (d)]. Moreover, a particle with speed $\bar{v}$ executes a circular orbit, if placed at a distance $r_{m}$ from the center, with $r_{m} \Omega\left(r_{m}\right)=\bar{v}$, and oriented counterclockwise [panel (e)]; upon inverting orientation, its orbit changes to an open, bound orbit with $r_{m} \leqslant r \leqslant r_{M}$, where $r_{M}$ satisfies the equality $\left[r_{M} A_{\phi}\left(r_{M}\right)-r_{m} A_{\phi}\left(r_{m}\right)\right] /\left(r_{M}+r_{m}\right)=\bar{v}$ [panel (f)]. More in general, for any choice of $p_{\phi}$ and $E$, one can always determine two radii, $r_{m}$ and $r_{M}$, such that the particle is trapped at any time with $r_{m} \leqslant r(t) \leqslant r_{M}$. Clearly, unbound radial diffusion is only possible in the presence of thermal fluctuations: the diffusing particle keeps exchanging energy with the heat bath on a time scale of the order of $m / \gamma$; its kinetic energy is exponentially distributed, $P(E)=e^{-E / k T} / k T$, with constant average $\langle E\rangle=k T$, whereas $p_{\phi}$ grows indefinitely. The question we address in the forthcoming sections is, then, how fast the particle diffuses out of the magnetic trap.

\section{NUMERICAL RESULTS}

We integrated the Langevin equations (1) for different damping regimes and radial profiles of the magnetic field, by numerically implementing a Euler-Maruyama algorithm. We set the integration time step not larger than $\Delta t=10^{-6}$, so as to make sure that during the entire simulation run it was much shorter than the (progressively decreasing) Larmor's period, that is $\Omega \Delta t \ll 1$. All stochastic averages reported below have been taken over 100 different realizations, which ensured an accurate fitting of the diffusion exponents and the relevant coefficients.

We focused on the radial diffusion of a particle initially at rest at the center of the trap, i.e., with $\mathbf{r}(0)=0$. The 


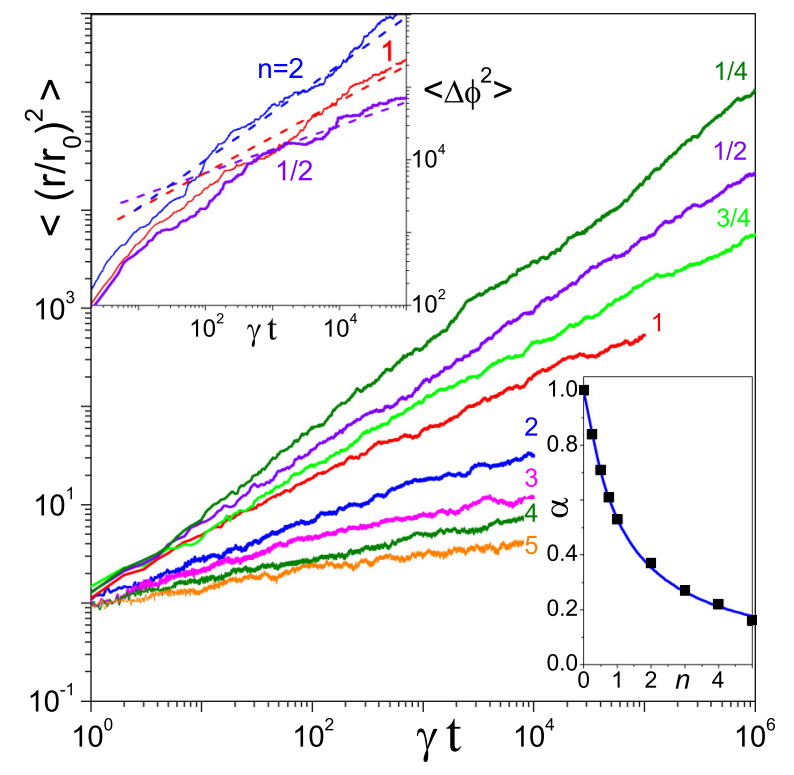

FIG. 2. (Color online) Particle diffusion $\left\langle\mathbf{r}^{2}(t)\right\rangle$ vs $t$ for the radially dependent $\Omega(r)$ of Eq. (2) with $\Omega_{0}=\Omega_{r}=1$ and positive $n$ (see legend). Lower-right inset: anomalous diffusion exponent. $n$ dependence of the fitting exponent $\alpha_{n}$ introduced in Eq. (3): simulation data (dots) vs our prediction, $\alpha_{n}=1 /(n+1)$, from Eq. (5) (solid curve). The dot $\alpha_{0}=1$ obtained in the limit $n \rightarrow 0$ (normal diffusion) has been added for completeness. Upper-left inset: the corresponding phase diffusion, $\langle\Delta \phi\rangle=\left\langle\phi^{2}\right\rangle-\langle\phi\rangle^{2}$ vs $t$, for three choices of $n$ (see legend); the relevant asymptotic laws, Eq. (6), are drawn for a comparison (dashed lines). Other simulation parameters are $q=m=1, k T=1, \gamma=0.1$, and $r(0)=0$.

particle m.s.d. thus reads $\left\langle\mathbf{r}^{2}(t)\right\rangle=\left\langle x^{2}(t)\right\rangle+\left\langle y^{2}(t)\right\rangle$, where $\langle\cdots\rangle$ denotes a stochastic average. Curves of $\left\langle\mathbf{r}^{2}(t)\right\rangle$ versus $t$ for different values of $n$ and $\gamma$ are displayed in Figs. 2-4. A few remarkable properties of the equilibrium diffusion process in magnetic traps with $n>0$ are apparent by inspection.

(i) For long simulation times, $\langle\mathbf{r}(t)\rangle$ vanishes no matter what the initial condition [see trajectory sampled in Fig. 1(b)], whereas $\left\langle\mathbf{r}^{2}(t)\right\rangle$ diverges asymptotically with time according to the subdiffusive power law,

$$
\left\langle\mathbf{r}^{2}(t)\right\rangle=D(n, \gamma) t^{\alpha_{n}}
$$

where the exponent $\alpha_{n}$ never exceeds unity, $\alpha_{n}<1$, and decreases with $n$, as shown in the inset of Fig. 2 . In the limit $n \rightarrow 0$ the normal diffusion exponent $\alpha_{0}=1$ is recovered, as expected (see lower-right inset of Fig. 2).

(ii) This instance of anomalous diffusion persists indefinitely with increasing the observation time. The curves plotted in Fig. 2 clearly indicate that the anomalous exponent $\alpha_{n}$ does not depend on $\gamma$. This property has been numerically confirmed over a wide $\gamma$ interval.

(iii) The coefficient $D(n, \gamma)$ scales with $\gamma$ also according to a simple power law, $D(n, \gamma)=D_{n} \gamma^{\beta_{n}}$, illustrated in panel (a) of Fig. 3. Similar to $\alpha_{n}$, the exponent $\beta_{n}$, too, is a decreasing function of $n$.

(iv) Normal diffusion with $\alpha_{n}=1$ was observed as a shorttime transient only for $\gamma \gg \Omega_{0}$ [see Figs. 4(a) and 4(b)]. This implies that, as the particle diffuses from the center to the
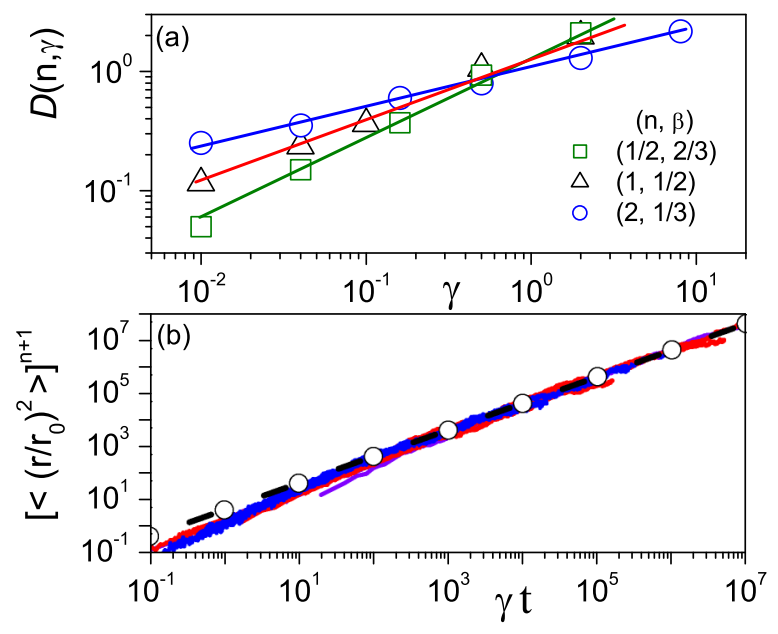

FIG. 3. (Color online) Dependence of the asymptotic diffusion law, $\left\langle\mathbf{r}^{2}(t)\right\rangle$ vs $t$, on $\gamma$ and $n$. (a) $\gamma$ dependence of the fitting parameter $D(n, \gamma)$ of Eq. (3) for different $n$ (see legend). The power laws $D(n, \gamma) \propto \gamma^{\beta_{n}}$ with $\beta_{n}=\alpha_{n}$ (straight lines) are drawn as a validity check of Eq. (5). (b) Rescaled diffusion curves, $\left\langle\mathbf{r}^{2}(t)\right\rangle^{n+1}$ vs $\gamma t$, for $n=1 / 2,1$, and 2 , and $\gamma=0.01,0.04,0.16,0.5$, and 2 . The color code is not reported as, after rescaling, the data sets are undistinguishable. For $\gamma t \gg 1$, all data sets collapse on top of the straight line with slope $\left[2 v_{\text {th }} /\left(\Omega_{r} r_{0}\right)\right]^{2}$ predicted in Eq. (5) (thick dotted-dashed line). The remaining simulation parameters are as in Fig. 2.

high-field periphery of the trap, anomalous diffusion inevitably sets on.

(v) When expressed in polar coordinates, $(r, \phi)$, the results of (i) imply that the particle distance $r(t)$ from the origin also diffuses anomalously, with $\langle r(t)\rangle^{2}$ and $\left\langle r^{2}(t)\right\rangle$ both being proportional to $\left\langle\mathbf{r}^{2}(t)\right\rangle$.

(vi) The polar angle $\phi$ also exhibits anomalous diffusion; its m.s.d., $\left\langle\Delta \phi^{2}(t)\right\rangle=\left\langle\phi^{2}(t)\right\rangle-\langle\phi(t)\rangle^{2}$, grows with time according to a power law with exponent $\gamma_{n}<1$ (Fig. 2, top-left inset), which, contrary to $\alpha_{n}$, increases with $n$. Note that the particle tends to circulate with net angular velocity oriented opposite to $\boldsymbol{\Omega}$. In short, the faster the radial diffusion, the slower the angular diffusion with the condition that the modulus of the mechanical angular momentum, $L_{z}=\dot{x} y-x \dot{y}$, grows in average with time like $t^{\alpha_{n} / 2}$ (not shown).

\section{SUBDIFFUSION}

We try now a quantitative interpretation of our numerical findings. In the bulk, i.e., in the absence of geometrical constrictions, the Brownian motion of a charged particle subject to a uniform magnetic field is characterized by the effective asymptotic diffusivity [22,23]

$$
D=D_{0} /\left(\gamma^{2}+\Omega^{2}\right),
$$

with $D_{0}$ defined in Eq. (1). Einstein's diffusion constant, $D_{0} / \gamma^{2}$, is recovered for $B=0$. The only effect of the magnetic field is to modify the relaxation rate of the diffusion process; the magnitude of such effect is measured by the ratio of the external field-controlled frequency, $\Omega(r)$, to the intrinsic medium viscous constant, $\gamma$. Since for long observation times $\left\langle\mathbf{r}^{2}(t)\right\rangle$ is bound to grow much larger than the square 

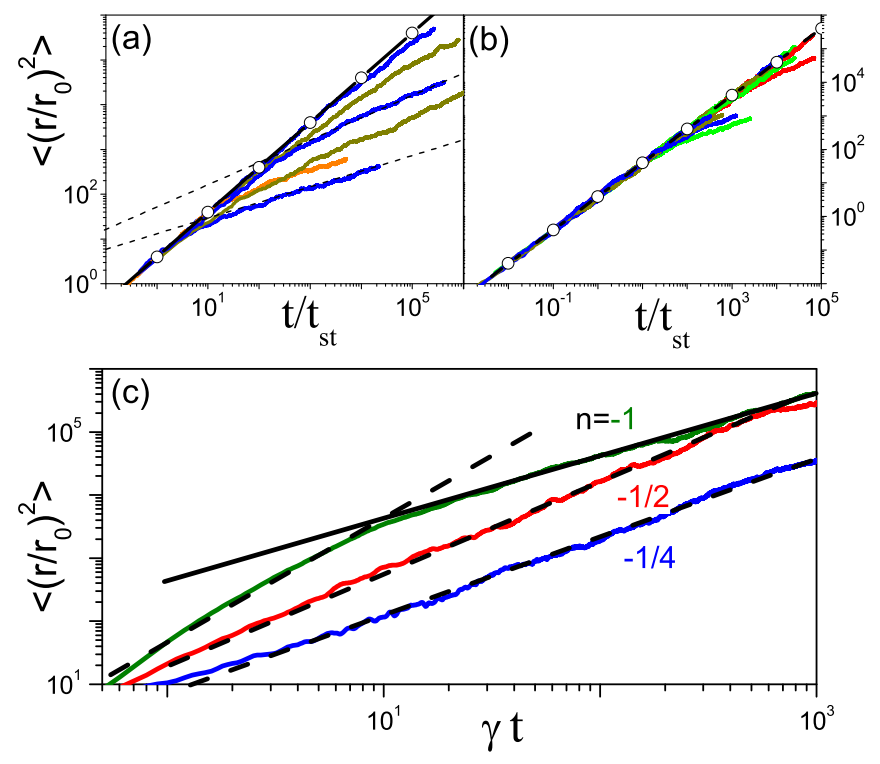

FIG. 4. (Color online) Diffusion transients $\left\langle\mathbf{r}^{2}(t)\right\rangle$ vs $t$ in units of $t_{\text {st }}=\gamma / \Omega_{0}^{2}$ for low (a) and large $\gamma(\mathrm{b}), \Omega_{0}=\Omega_{r}=1$, and $n=1 / 2,1$, and 2. Color code in panel (a): $\gamma=8$ (yellow), 24 (blue), and 100 (orange); for curves of the same color, $n$ decreases from top to bottom; for $n=2$ we only drew the orange curve and not the yellow one. In panel (b) $\gamma$ ranges between 100 and 1600; after rescaling the data sets are hardly distinguishable. In both panels the power law (7) for shorttime normal diffusion is represented by a thick dotted-dashed line with slope $\left[2 v_{\text {th }} /\left(\Omega_{0} r_{0}\right)\right]^{2}$. In panel (a) the asymptotic laws (5) for $\gamma=24$ and $n=1$ and $1 / 2$ are drawn for reader convenience. Panel (c): negative field radial gradients, $n<0,\left\langle\mathbf{r}^{2}(t)\right\rangle$ vs $t$, in dimensionless units, for $r(0)=1, \Omega_{0}=0, \Omega_{r}=1, \gamma=0.1$, and different $n$ (see legends). The transient law (8) is fitted to the data in the appropriate time domains (dashed lines); the expected asymptotic diffusion law is drawn as a solid line. The remaining simulation parameters are as in Fig. 2.

of the trapping length, $r_{0}^{2}$, the radial function $\Omega(r)$ can be approximated by $\Omega_{r}\left(r / r_{0}\right)^{n}$. Therefore, in the same limit, the diffusion process is dominated by $\Omega(r)$ rather than $\gamma$, that is, $D(r) \simeq D_{0} / \Omega^{2}(r)$. The m.s.d. of a Brownian particle with homogeneous diffusivity, $D$, would grow linearly with time, according to the standard diffusion law $\left\langle\mathbf{r}^{2}(t)\right\rangle=4 D t$. Here, however, the diffusivity depends on $r$ and, therefore, gets suppressed as the diffusion process advances in time. In view the above approximations, $D(t) \simeq D_{0} /\left[\Omega_{r}^{2}\left(\left\langle\mathbf{r}^{2}(t)\right\rangle / r_{0}^{2}\right)^{n}\right]$. On inserting this estimate of $D(t)$ in the standard diffusion equation for $\left\langle\mathbf{r}^{2}(t)\right\rangle$ and solving with respect to $\left\langle\mathbf{r}^{2}(t)\right\rangle$, one obtains the phenomenological asymptotic diffusion law

$$
\left\langle\mathbf{r}^{2}(t)\right\rangle / r_{0}^{2}=D_{n}^{(r)}(\gamma t)^{\frac{1}{n+1}},
$$

with $D_{n}^{(r)}=\left[2 v_{\text {th }} /\left(\Omega_{r} r_{0}\right)\right]^{\frac{2}{n+1}}$. Based on this simple heuristic argument, one expects that the exponents $\alpha_{n}$ and $\beta_{n}$ reported in Figs. 2 and 3 coincide as $\alpha_{n}=\beta_{n}$ and $\alpha_{n}=1 /(n+1)$. Moreover, the temperature dependence is accounted for by coefficient $D_{n}^{(r)}$ and suppressed for strong radial field gradients, $n \gg 1$. Both conclusions are well supported by our numerical data. In particular, Eq. (5) fits closely all the $\left\langle\mathbf{r}^{2}(t)\right\rangle$ curves plotted in Fig. 3(b) for different values of $n$ and $\gamma$.
The corresponding asymptotic law for the angular diffusion is immediately related to Eq. (5). From the phenomenological definition of tangential diffusion, $\left\langle\Delta \phi^{2}(t)\right\rangle\left\langle r^{2}(t)\right\rangle=$ $2\left(v_{\mathrm{th}}^{2} / \gamma\right) t$, it follows immediately that for $r \gg r_{0}$

$$
\left\langle\Delta \phi^{2}(t)\right\rangle=D_{n}^{(\phi)}(\gamma t)^{\gamma_{n}}
$$

with $D_{n}^{(\phi)}=2\left[v_{\text {th }} /\left(\gamma r_{0}\right)\right]^{2} / D_{n}^{(r)}$ and $\alpha_{n}+\gamma_{n}=1$, that is $\gamma_{n}=$ $n /(n+1)$. This prediction compares well with the simulation data for $\left\langle\Delta \phi^{2}(t)\right\rangle$; see top-left inset of Fig. 2.

The asymptotic power law (5) for $\left\langle\mathbf{r}^{2}(t)\right\rangle$ may be preceded by persistent diffusion transients. For short observation times, more precisely, as long as $\left\langle r^{2}(t)\right\rangle \ll r_{0}^{2}$, the radial dependence of the cyclotron frequency does not enter into play, as $\Omega(r) \simeq \Omega_{0}$. As a consequence, in the overdamped regime, $\gamma \gg \Omega_{0}$, the effective diffusivity of Eq. (4) is insensitive to the magnetic field, namely, $D \simeq D_{0} / \gamma^{2}=v_{\text {th }}^{2} / \gamma$. A particle injected in the vicinity of the center of the trap, $r(0) \ll r_{0}$, thus starts diffusing like

$$
\left\langle\mathbf{r}^{2}(t)\right\rangle_{t} / r_{0}^{2}=D_{\text {st }} \gamma t
$$

with $D_{\text {st }}=\left[2 v_{\text {th }} /\left(\gamma r_{0}\right)\right]^{2}$ independent of $n$. This explains the normal diffusion transients reported in Figs. 4(a) and 4(b). Moreover, we observed that particles injected at a finite distance, $r(0)$, from the center, initially diffuse slower than predicted in Eq. (7). This result is also consistent with the argument already used to derive Eq. (5). Therefore, as apparent in Fig. 4, the anomalous asymptotic diffusion law (5) applies regardless of the initial conditions, whereas transient diffusion laws do not.

The crossover between short-time normal diffusion and asymptotic anomalous diffusion with exponent $\alpha_{n}$ occurs at a crossover time $t_{c}$, which can also be estimated through our heuristic argument. Based on that argument, the onset of anomalous diffusion for $r(t)$ requires that $\Omega(r) \simeq$ $\Omega_{r}\left(r / r_{0}\right)^{n} \gg \gamma$. On making use of the diffusion law (5) it becomes apparent that anomalous diffusion can only occur for $t \gg t_{c}$ with $t_{c} \propto \gamma^{1+2 / n}$. This prediction is in qualitative agreement with our numerical findings, according to which $t_{c}$ increases with increasing $\gamma$ and lowering $n$.

The energetic interpretation of the anomalous diffusion phenomenon reported here is intuitively simple. As the particle diffuses far away from the trap center, its average Larmor radius grows very small, $R_{\Omega} \sim v_{\text {th }} / \Omega(r)$ [see also Fig. 1(b)], until, for $\Omega \gg \gamma / m$, the particle performs many free gyrations during the inertial time scale $m / \gamma$. Such a kinematic mechanism tends to suppress the effective particle diffusivity, as suggested by Eq. (4). Vice versa, when the heat bath causes the particle's speed to drop below $v_{\text {th }}$, the instantaneous curvature radius grows larger and the particle may diffuse longer distances during the same time scale. Thus, as time progresses, the diffusion process becomes increasingly sensitive to the fluctuations of the kinetic energy around its average, $k T$; hence the subdiffusive values of the exponent $\alpha, \alpha<1$. Note that, in the absence of thermal fluctuations, the particle would be confined within a maximal distance from the center, $r_{M}$, which would depend on its kinetic energy (see Sec. II). 


\section{SUPERDIFFUSIVE TRANSIENTS}

Remarkable diffusion transients have been detected for the not uncommon case of a magnetic field with negative radial gradient. For instance, the magnetic field felt by a charged particle diffusing in a plane perpendicular to a magnetic tip typically decreases with increasing its distance from the tip. This situation can be modeled by setting $\Omega_{0}=0$ and $n<0$ in Eq. (2) for $\Omega(r)$. Of course, in the asymptotic limit $\Omega(r)$ grows negligibly small with respect to $\gamma$, so that the corresponding asymptotic diffusion law formally coincides with Eq. (7), that is $\left\langle\mathbf{r}^{2}(t)\right\rangle=4\left(v_{\mathrm{th}}^{2} / \gamma\right) t$. To analyze the diffusion transients we notice that at shorter times, as long as $\Omega(r) \gg \gamma$, the effective diffusion coefficient of Eq. (3) increases with time, that is $D(t) \simeq\left(D_{0} / \Omega_{r}^{2}\right)\left[\left\langle\mathbf{r}^{2}(t)\right\rangle / r_{0}^{2}\right]^{|n|}$, where for $\left\langle\mathbf{r}^{2}(t)\right\rangle$ we must now use the known asymptotic power law given above. Accordingly, during the initial stage of the diffusion process, $\left\langle\mathbf{r}^{2}(t)\right\rangle_{t} \simeq 4 D(t) t$, which leads to the superdiffusive transient law

$$
\left\langle\mathbf{r}^{2}(t)\right\rangle_{t} / r_{0}^{2}=D_{\mathrm{st}}(\gamma t)^{1+|n|},
$$

with $D_{\text {st }}=\left(\gamma / \Omega_{r}\right)^{2}\left[2 v_{\text {th }} /\left(\gamma r_{0}\right)\right]^{2(1+|n|)}$. In Fig. 4(c) Eq. (8) was used as a fitting law to prove the correctness of the predicted transient exponents for $-1<n<0$. For $n<-1$ (not shown) our derivation of Eq. (8) is no longer tenable as $\Omega(r)$ grows smaller than $\gamma$ so fast that the particle dynamics is practically insensitive to the magnetic field; vice versa, on lowering $|n|$, the agreement with the numerical data improves. Most remarkably, for weakly decaying $B(r)$ the superdiffusive transient can span several time decades before normal diffusion sets on.

[1] D. Bohm, E. H. S. Burhop, and H. S. W. Massey, in Characteristics of Electrical Discharges in Magnetic Fields, edited by A. Guthrie and R. K. Wakcrling (MacGraw-Hill, New York, 1949); K. H. Geissler, Phys. Rev. 171, 179 (1968); J. B. Tayor and B. McNamara, Phys. Fluids 14, 1492 (1971).

[2] F. F. Chen, Introduction to Plasma Physics and Controlled Fusion, 2nd ed., Plasma Physics Vol. I (Plenum, New York, 1984).

[3] A. V. Bobylev, F. A. Maao, A. Hansen, and E. H. Hauge, Phys. Rev. Lett. 75, 197 (1995).

[4] J. Haus and K. Kehr, Phys. Rep. 150, 263 (1987).

[5] J.-P. Bouchaud and A. Georges, Phys. Rep. 195, 127 (1990).

[6] J. Wagenhuber, T. Geisel, P. Niebauer, and G. Obermair, Phys. Rev. B 45, 4372 (1992).

[7] P. Tierno, T. H. Johansen, and T. M. Fischer, Phys. Rev. Lett. 99, 038303 (2007).

[8] C. Oleksy, Phys. Rev. E 59, 3864 (1999) and references therein.

[9] F. Otsuka and T. Hada, Prog. Theor. Phys. Suppl. 165, 204 (2006).

[10] W. H. Matthaeus, G. Qin, J. W. Bieber, and G. P. Zank, Astrophys. J. Lett. 590, L53 (2003); G. M. Webb, G. P. Zank, E. Kh. Kaghashvili, and J. A. le Roux, Astrophys. J. 651, 211 (2006).

[11] B. A. Carreras, V. E. Lynch, and G. M. Zaslavsky, Phys. Plasmas 8, 5096 (2001).

\section{CONCLUSION}

In conclusion, we have proved that a simple twodimensional driven Hamiltonian system coupled to an equilibrium heat bath, modeled by a Stokes' friction term and a Gaussian $\delta$-correlated noise, may undergo anomalous diffusion. A key condition is that the two degrees of freedom are not separable [24] and, more precisely, their coordinates and velocities are nonlinearly coupled. If such coupling vanishes as diffusion progresses, then anomalous diffusion is restricted to an appropriate superdiffusive transient; in the opposite limit, the nonlinear coupling grows stronger and the ensuing asymptotic diffusion regime is subdiffusive. Direct observation of these effects is conceivable on very different spatial scales, from galactic distances (cosmic rays) down to molecular lengths (magnetic tweezers). In particular, Bohm diffusion across an inhomogeneous distribution of field lines could be anomalous, with exponent not equal to one, for rather long observation times. At this point one might still wonder whether an inhomogeneous magnetic field can effectively suppress the cross-field diffusion of a charged particle [10]. Following the heuristic argument used throughout this paper, we immediately see that in the presence of exponential radial gradients of the field, the particle m.s.d. diverges logarithmically. This means that diffusion is critically slowed down and the particle may take an indefinitely long time to cross the trapping magnetic-field lines.

\section{ACKNOWLEDGMENTS}

S.E.S. acknowledges support from the Leverhulme Trust; F.M. acknowledges support from the European Commission, under the Seventh Framework Programme (256959, NanoPower).

[12] T. J. M. Boyd and J. J. Sanderson, The Physics of Plasma (Cambridge University Press, Cambridge, UK, 2003).

[13] V. L. Ginzburg, V. A. Dogiel, V. S. Berezinsky, S. V. Bulanov, and V. S. Ptuskin, Astrophysics of Cosmic Rays (North-Holland, Amsterdam, 1990).

[14] K. C. Neuman and A. Nagy, Nat. Methods 5, 491 (2008).

[15] Y. Meroz, I. M. Sokolov, and J. Klafter, Phys. Rev. Lett. 107, 260601 (2011); F. Thiel, F. Flegel, and I. M. Sokolov, ibid. 111, 010601 (2013).

[16] R. Metzler and J. Klafter, Phys. Rep. 339, 1 (2000).

[17] J. Kärger and M. Ruthven, Diffusion in Zeolites and in Other Microporous Solids (Wiley, New York, 1992).

[18] D. ben Avraham and S. Havlin, Diffusion and Reactions in Fractals and Disordered Systems (Cambridge University Press, Cambridge, UK, 2000).

[19] G. M. Zaslavsky, Hamiltonian Chaos and Fractional Dynamics (Oxford University Press, Oxford, 2005).

[20] R. Metzler, E. Barkai, and J. Klafter, Phys. Rev. Lett. 82, 3563 (1999).

[21] T. P. Simões and R. E. Lagos, Physica A 355, 274 (2005); J. I. Jiménez-Aquino and M. Romero-Bastida, Phys. Rev. E 76, 021106 (2007).

[22] J. B. Taylor, Phys. Rev. Lett. 6, 262 (1961).

[23] B. Kurşunoğlu, Phys. Rev. 132, 21 (1963).

[24] A. Pototsky, F. Marchesoni, F. V. Kusmartsev, P. Hänggi, and S. E. Savel'ev, Eur. Phys. J. B 85, 356 (2012). 